\title{
Nonequilibrium dynamics of the complex Ginzburg-Landau equation: Analytical results
}

\author{
Subir K. Das, ${ }^{1}$ Sanjay Puri, ${ }^{1}$ and M. C. Cross $^{2}$ \\ ${ }^{1}$ School of Physical Sciences, Jawaharlal Nehru University, New Delhi 110067, India \\ ${ }^{2}$ Department of Physics, California Institute of Technology, Pasadena, California 91125
}

(Received 15 March 2001; published 20 September 2001)

\begin{abstract}
We present a detailed analytical and numerical study of nonequilibrium dynamics for the complex GinzburgLandau equation. In particular, we characterize evolution morphologies using spiral defects. This paper is the first in a two-stage exposition. Here, we present analytical results for the correlation function arising from a single-spiral morphology. We also critically examine the utility of the Gaussian auxiliary field ansatz in characterizing a multispiral morphology. In the next paper of this exposition we will present detailed numerical results.
\end{abstract}

DOI: 10.1103/PhysRevE.64.046206

PACS number(s): 64.60.Cn, 05.70.Ln

\section{INTRODUCTION}

There have been many studies of pattern formation in the complex Ginzburg-Landau (CGL) equation, which has the general form

$$
\begin{aligned}
\frac{\partial \psi(\vec{r}, t)}{\partial t}= & \psi(\vec{r}, t)+(1+i \alpha) \nabla^{2} \psi(\vec{r}, t) \\
& -(1+i \beta)|\psi(\vec{r}, t)|^{2} \psi(\vec{r}, t) .
\end{aligned}
$$

In Eq. (1), $\psi(\vec{r}, t)$ is a complex order-parameter field which depends on space $(\vec{r})$ and time $(t)$; and $\alpha, \beta$ are real parameters. The CGL equation arises in diverse contexts, e.g., chemical oscillations [1], thermal convection in binary fluids [2], multimode lasers [3], etc. An overview of applications of the CGL equation is provided in the review article by Cross and Hohenberg [4]. The importance of the CGL equation stems from the fact that it provides a generic description of the slow modulation of oscillations in a spatially extended system near a Hopf bifurcation [5].

The CGL equation exhibits a rich range of dynamical behavior with variation of the parameters $\alpha$ and $\beta$, and the "phase diagram" has been investigated (mostly numerically) by various authors [6]. In a large range of parameter space, the emergence and interaction of spiral (and antispiral) defects play an important role in determining the morphology. Our present work focuses on characterizing pattern formation in the CGL equation using spiral-defect structures.

We have studied nonequilibrium dynamics analytically and numerically in the CGL equation with $\alpha=0$. Typically, we consider the evolution morphology resulting from a small-amplitude random initial condition. There has been intense research interest in such problems in the context of far-from-equilibrium statistical physics-for reviews, see $[7,8]$. The simplest problem in this class considers a homogeneous two-phase mixture, which has been rendered thermodynamically unstable by a rapid quench below the critical coexistence temperature. An example of such a system is a two-state ferromagnet (in zero magnetic field) at high temperatures, which consists of a homogeneous mixture of "up" and "down" spins. However, below the critical tem- perature, the system prefers to be in a spontaneously magnetized state. The evolution of the system from the unstable initial state is a complex nonlinear process. In appropriate dimensionless units, this evolution is described by the timedependent Ginzburg-Landau (TDGL) equation, i.e., Eq. (1) with $\psi(\vec{r}, t)$ real and $\alpha=\beta=0$. The system evolves by the formation and growth of domains that are enriched in either up or down spins and are characterized by a time-dependent length scale $L(t)$. In the case of a pure and isotropic ferromagnet, the domain growth law is $L(t) \sim t^{1 / 2}$, which is referred to as the Lifshitz-Cahn-Allen (LCA) law [9]. The primary mechanism for domain coarsening (or "phase-ordering dynamics") is the curvature-driven motion and annihilation of interfaces (or defects). Ohta et al. [10] have formulated an interface-dynamics approach to obtain an analytic form for the equal-time correlation function of a phase-ordering ferromagnet.

Next, let us consider the dynamical $X Y$ model, which is Eq. (1) with $\psi(\vec{r}, t)$ complex but $\alpha=\beta=0$. In this case, the relevant defect structures (for dimensionality $d \geqslant 2$ ) are vortices (or vortex lines, etc.), and domain growth is driven by the motion and annihilation of vortices and antivortices. Puri [11] obtained the time-dependent correlation function for the $X Y$ model, using singular-perturbation methods due to Suzuki [12], Kawasaki et al. [13], and Puri and Roland [14]. Furthermore, Bray and Puri [15] and (independently) Toyoki [16] obtained the time-dependent correlation function for the vector TDGL equation with $O(n)$ symmetry in $d$ dimensions when $n \leqslant d$, i.e., when topological defects are present. [The dynamical $X Y$ model corresponds to the case with $O(2)$ symmetry.] The corresponding domain growth law is again the LCA law $L(t) \sim t^{1 / 2}$, with logarithmic corrections when $n=d[8,17]$. To the best of our knowledge, there are no general results available for the case with $n>d$, where the absence of topological defects makes it difficult to characterize the dynamical evolution.

The present two-stage exposition focuses on phaseordering dynamics in the CGL equation with $\alpha=0$. Furthermore, the analytical and numerical results presented here are for the two-dimensional case, where spirals are point defects. However, the analytical results obtained by us can easily be extended to the case with $\alpha \neq 0$ and $d \geqslant 2$, as the underlying 
paradigm remains the same, i.e., spiral defects still determine the morphology in large regions of parameter space and for higher dimensionality.

Following the work of Hagan [18], Aranson et al. [19], and Chate and Manneville [6], we briefly discuss the phase diagram of the $d=2$ CGL equation with $\alpha=0$. The limit $\beta$ $=0$ corresponds to the dynamical $X Y$ model, which is well understood $[11,8]$. Without loss of generality, we consider the case with $\beta \geqslant 0$. For $0 \leqslant \beta \leqslant \beta_{1}\left(\beta_{1} \simeq 1.397\right.$ [18]), spirals (which are asymptotically plane waves) are linearly stable to fluctuations. For $\beta_{1}<\beta \leqslant \beta_{2}\left(\beta_{2} \simeq 1.82[19,6]\right)$, spirals are linearly unstable to fluctuations, but the growing fluctuations are advected away, i.e., the spiral structure is globally stable. Finally, for $\beta_{2}<\beta$, the spirals are globally unstable structures and cannot exist for extended times [19]. Our results correspond to the parameter regime with $\beta \leqslant \beta_{2}$.

In this paper we present analytical studies of the correlation function resulting from single-spiral and multispiral morphologies. A later paper will present detailed numerical results and compare them with the analytical results presented here. This paper is organized as follows. In Sec. II, we obtain analytical results for the correlation function of a single-spiral morphology. In Sec. III, we critically examine the utility of the Gaussian auxiliary field (GAF) ansatz [8] for the characterization of a multispiral morphology. Section IV concludes this paper with a brief summary and discussion of our analytical results.

\section{CORRELATION FUNCTION FOR A SINGLE-SPIRAL MORPHOLOGY}

Figure 1 shows a typical evolution from a smallamplitude random initial condition for the $d=2$ CGL equation with $\alpha=0$ and $\beta=1$. We have plotted constant-phase regions in this figure, and it is clear that the evolving morphology is characterized by spirals and their interactions. (We use the term "spiral" for both spirals and antispirals, unless specifically stated otherwise.) There is a characteristic length scale, e.g., interspiral spacing or square root of inverse defect density, which we denote as $L$. Details of our simulation techniques and comprehensive numerical results will be provided in a future paper. Figure 1 is shown here only to motivate our subsequent discussion.

We would like to quantitatively characterize the evolution morphology shown in Fig. 1. The standard tool for this is the correlation function of the order-parameter field [7,8], which we will define shortly. (The momentum-space structure factor is obtained as the Fourier transform of the real-space correlation function.) At the simplest level of approximation, the morphology in the frames of Fig. 1 can be interpreted as consisting of disjoint spirals, each of size $L$. (Of course, this overlooks modulations of the order-parameter field at spiralspiral boundaries, but we will discuss that later.) Therefore, it is obviously of relevance to compute the correlation function for a single-spiral solution.

The CGL equation with $\alpha=0$ has been studied by Hagan [18], who found that there is a family of spiral solutions with the following functional form (in $d=2$ ):
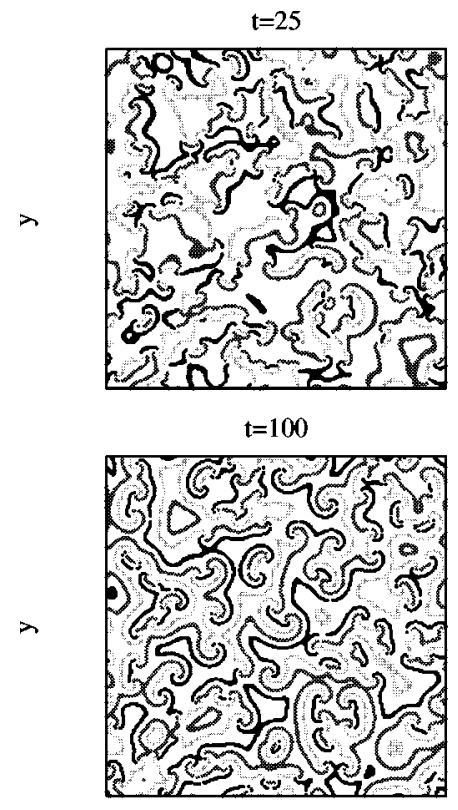

$\mathbf{x}$

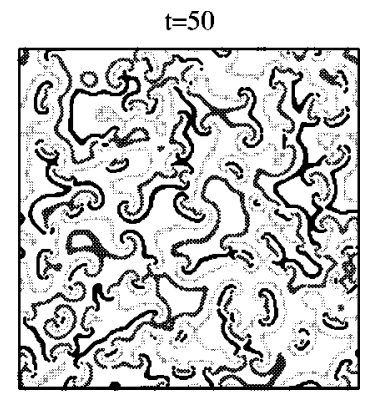

$\mathrm{t}=1000$

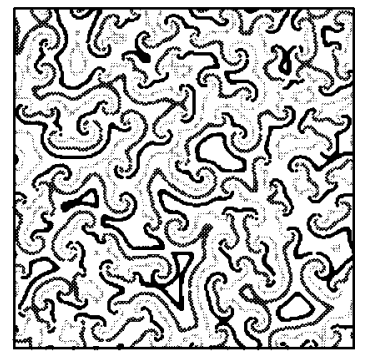

$\mathbf{x}$
FIG. 1. Evolution of a small-amplitude random initial condition for the complex Ginzburg-Landau equation with $\alpha=0, \beta=1$. These evolution pictures were obtained from an isotropic Euler discretization of Eq. (1), implemented on an $N^{2}$ lattice $(N=256)$ with periodic boundary conditions in both directions. The discretization mesh sizes were $\Delta t=0.01$ and $\Delta x=1.0$. The pictures show regions of constant phase $\theta_{\psi}=\tan ^{-1}(\operatorname{Im} \psi / \operatorname{Re} \psi)$, measured in radians, with the following coding: $\theta_{\psi} \in[1.85,2.15]$ (black); $\theta_{\psi}$ $\in[3.85,4.15]$ (dark gray); $\theta_{\psi} \in[5.85,6.15]$ (light gray). The snapshots are labeled by the appropriate evolution times.

$$
\psi(\vec{r}, t)=\rho(r) \exp \left[-i \beta\left(1-q^{2}\right) t+i m \theta-i \phi(r)\right],
$$

where $\vec{r} \equiv(r, \theta), q \geqslant 0$ is a constant which is determined uniquely as a function of $\beta$, and $m$ is the number of arms in the spiral. The cases with $m>0$ and $m<0$ correspond to a spiral and antispiral, respectively. The limiting forms of the functions $\rho(r)$ and $\phi(r)$ are

$$
\begin{gathered}
\rho(r) \rightarrow\left(1-q^{2}\right)^{1 / 2}, \quad \phi^{\prime}(r) \rightarrow q \quad \text { as } r \rightarrow \infty, \\
\rho(r) \rightarrow a r^{m}, \quad \phi^{\prime}(r) \rightarrow r \quad \text { as } r \rightarrow 0,
\end{gathered}
$$

where the constant $a$ is determined by finiteness conditions. Hagan presented explicit solutions for $q(\beta)$ in the cases with $m=1,2$. We will focus on the case with $m= \pm 1$, as only the one-armed spirals are expected to be stable in the evolution [18]. Figure 2 plots Hagan's solution for $q(\beta)$ (with $\beta$ $\leqslant 1.5$ ) in the case with $m= \pm 1$. In the simple limit $\beta=0$, we have $q=0$, and the spiral solution simplifies to the vortex solution-for the $m= \pm 1$ vortex, the lines of constant phase correspond to constant $\theta$. Spiral solutions for the general case with $\alpha, \beta \neq 0$ were discussed by Aranson et al. $[19,20]$.

We are interested in the correlation function for a onearmed spiral at large length scales, so we simplify Eq. (2) as

$$
\psi(\vec{r}, t) \simeq \sqrt{1-q^{2}} \exp \left[-i \beta\left(1-q^{2}\right) t+i(\theta-q r)\right],
$$


where we have specialized to the $m=1$ case. The correlation between points $\vec{r}_{1}$ and $\vec{r}_{2}$ is determined as

$$
\begin{aligned}
C\left(\vec{r}_{1}, \vec{r}_{2}, t\right) & =\operatorname{Re}\left\{\psi\left(\vec{r}_{1}, t\right) \psi^{*}\left(\vec{r}_{2}, t\right)\right\} \\
& \simeq\left(1-q^{2}\right) \operatorname{Re}\left\{\exp \left[i\left(\theta_{1}-q r_{1}\right)-i\left(\theta_{2}-q r_{2}\right)\right]\right\} \\
& \equiv C\left(\vec{r}_{1}, \vec{r}_{2}\right) .
\end{aligned}
$$

The average correlation function is obtained by integrating over the point $\vec{r}_{1}$, setting $\vec{r}_{2}=\vec{r}_{1}+\vec{r}_{12}$, i.e.,

$$
\begin{aligned}
C\left(r_{12}\right)= & \frac{1}{V} \int d \vec{r}_{1} C\left(\vec{r}_{1}, \vec{r}_{1}+\vec{r}_{12}\right) h\left(L-\left|\vec{r}_{1}+\vec{r}_{12}\right|\right) \\
= & \frac{\left(1-q^{2}\right)}{V} \operatorname{Re} \int d \vec{r}_{1} \exp \left[i \left(\theta_{1}-\theta_{2}-q r_{1}\right.\right. \\
& \left.\left.+q\left|\vec{r}_{1}+\vec{r}_{12}\right|\right)\right] h\left(L-\left|\vec{r}_{1}+\vec{r}_{12}\right|\right),
\end{aligned}
$$

where $V$ is the spiral volume. In Eq. (6), we use the step function $h(x)=1(0)$ if $x \geqslant 0(x<0)$, which ensures that we do not include points that lie outside the defect of size $L$.

For $d=2$, the vector notation $\vec{r}_{2}=\vec{r}_{1}+\vec{r}_{12}$ is equivalent to $r_{2} e^{i \theta_{2}}=r_{1} e^{i \theta_{1}}+r_{12} e^{i \theta_{12}}$. Thus we have

$$
e^{i \theta_{2}}=\frac{r_{1} e^{i \theta_{1}}+r_{12} e^{i \theta_{12}}}{\left[r_{1}^{2}+r_{12}^{2}+2 r_{1} r_{12} \cos \left(\theta_{1}-\theta_{12}\right)\right]^{1 / 2}},
$$

and

$$
\begin{aligned}
C\left(r_{12}\right)= & \frac{\left(1-q^{2}\right)}{V} \operatorname{Re} \int_{0}^{L} d r_{1} r_{1} \int_{0}^{2 \pi} d \theta_{1} \\
& \times \frac{r_{1}+r_{12} e^{i\left(\theta_{1}-\theta_{12}\right)}}{\left[r_{1}^{2}+r_{12}^{2}+2 r_{1} r_{12} \cos \left(\theta_{1}-\theta_{12}\right)\right]^{1 / 2}} \\
& \times \exp \left(-i q\left\{r_{1}-\left[r_{1}^{2}+r_{12}^{2}+2 r_{1} r_{12}\right.\right.\right. \\
& \left.\left.\left.\times \cos \left(\theta_{1}-\theta_{12}\right)\right]^{1 / 2}\right\}\right) h\left(L-\left|\vec{r}_{1}+\vec{r}_{12}\right|\right) .
\end{aligned}
$$

We introduce the variables $\theta_{1}-\theta_{12}=\theta ; x=r_{1} / L ; r=r_{12} / L$, to obtain

$$
\begin{aligned}
C\left(r_{12}\right)= & \frac{\left(1-q^{2}\right)}{\pi} \operatorname{Re} \int_{0}^{1} d x x \int_{0}^{2 \pi} d \theta \\
& \times \frac{x+r e^{i \theta}}{\left(x^{2}+r^{2}+2 x r \cos \theta\right)^{1 / 2}} \\
& \times \exp \left[-i q L\left\{x-\left(x^{2}+r^{2}+2 x r \cos \theta\right)^{1 / 2}\right\}\right] \\
& \times h\left[1-\left(x^{2}+r^{2}+2 x r \cos \theta\right)^{1 / 2}\right],
\end{aligned}
$$

where we have used $V=\pi L^{2}$ in $d=2$. Thus, the scaling form of the single-spiral correlation function is $C\left(r_{12}\right) / C(0)$ $\equiv g\left(r_{12} / L, q^{2} L^{2}\right)$. In general, there is no scaling with the spiral size because of the additional factor $q L$. We recover scaling only in the limit $q=0(\beta=0)$, which corresponds to the case of a vortex. Essentially, spirals of different sizes are

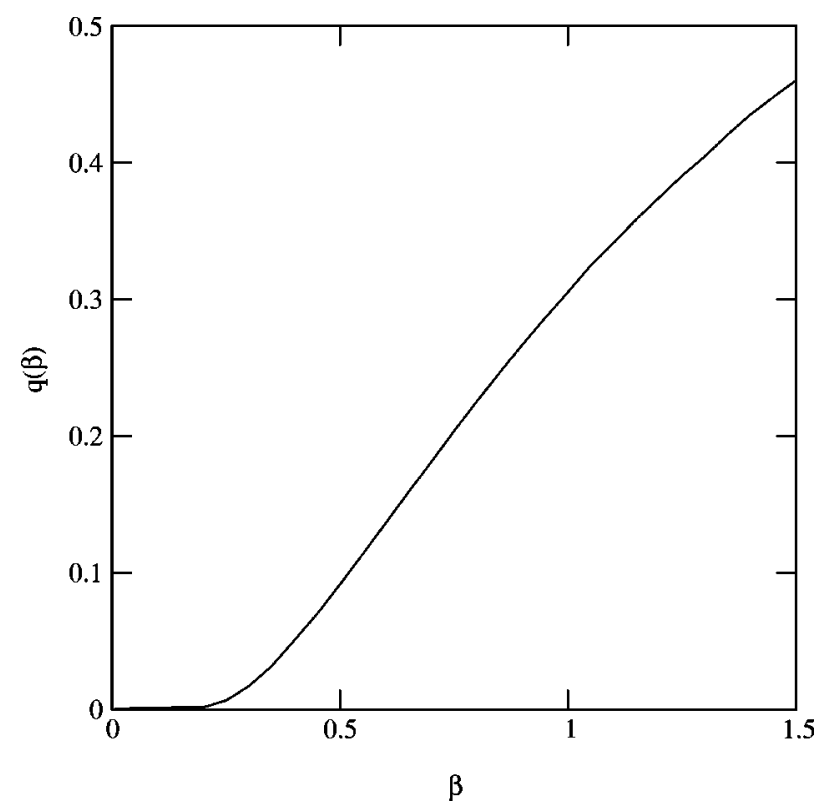

FIG. 2. Plot of $q(\beta)$ vs $\beta$ for the one-armed spiral solution of the CGL equation with $\alpha=0$. (Compare Fig. 5 of Ref. [18].)

not morphologically equivalent because there is more rotation in the phase as one goes out further from the core.

Figure 3 plots $C\left(r_{12}\right) / C(0)$ vs $r_{12} / L$ for the case with $\beta=1(q \simeq 0.306)$. These results are obtained by a direct numerical integration of Eq. (9). We consider four different values of $L$. The functional form in Fig. 3 exhibits nearmonotonic behavior for small values of $L$ (i.e., in the vortex limit); and pronounced oscillatory behavior for larger values of $L$, as is expected from the integral expression. Notice that $r_{12} / L \leqslant 2$-larger values of $r_{12}$ correspond to the point $\vec{r}_{2}$ lying outside the defect.

Before we proceed, we should point out that the imaginary part of the integral in Eq. (9) is nonzero, in generalcorresponding to a weak correlation between the real and imaginary parts of the order-parameter field. The imaginary part can also be obtained with relative ease. However, we will confine our discussion to the conventional definition of the correlation function in Eq. (5). Let us next consider the asymptotic behavior of the correlation function in the limit $r_{12} / L \rightarrow 0$, although $r_{12}$ is still much larger than the size of the defect core $\xi$.

\section{A. Case with $\boldsymbol{\beta}=0$}

In the case with $\beta=0$, we have $q=0$ and the integral expression in Eq. (9) simplifies as

$$
\begin{aligned}
C\left(r_{12}\right)= & \frac{1}{\pi} \operatorname{Re} \int_{0}^{1} d x x \int_{0}^{2 \pi} d \theta \frac{x+r e^{i \theta}}{\left(x^{2}+r^{2}+2 x r \cos \theta\right)^{1 / 2}} \\
& \times h\left[1-\left(x^{2}+r^{2}+2 x r \cos \theta\right)^{1 / 2}\right] .
\end{aligned}
$$

The behavior in the $r \rightarrow 0$ limit is of considerable interest as it determines the large-wave-vector $(k \rightarrow \infty)$ behavior of the structure factor [8]. In that case, we can neglect the step function on the right-hand side (RHS) of Eq. (10) as it only 


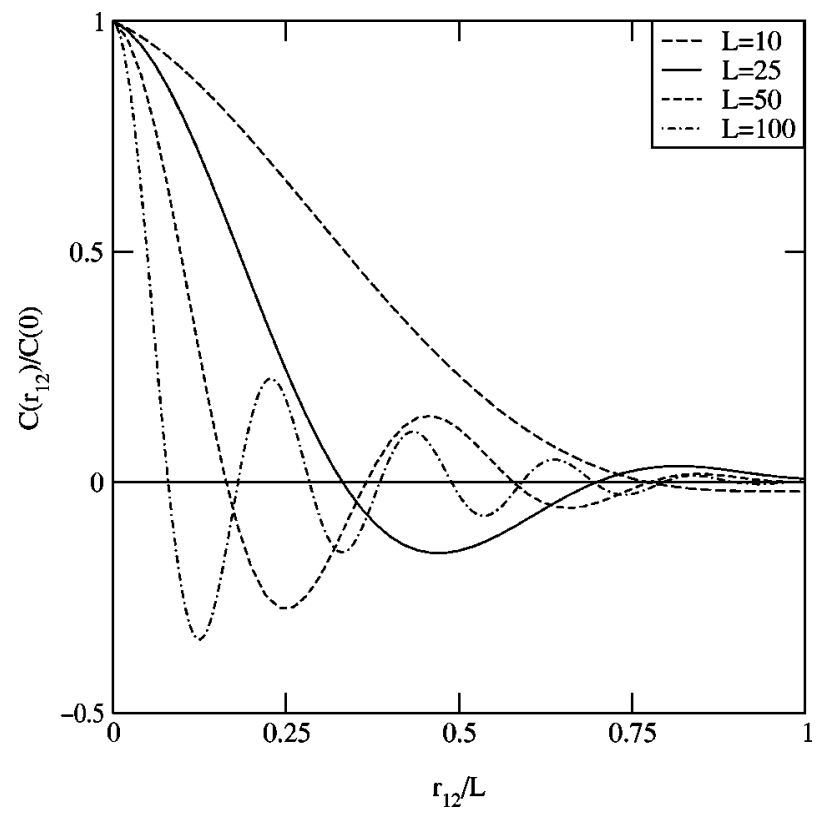

FIG. 3. Correlation function for the one-armed spiral solution when $\beta=1 \quad(q \simeq 0.306)$. We plot $C\left(r_{12}\right) / C(0)$ vs $r_{12} / L$ for different spiral sizes $L=10,25,50,100$ - denoted by the specified line types. The results are obtained from a direct numerical integration of Eq. (9).

provides corrections at the edge of the vortex defect. Then, after some algebra, we obtain the result

$$
C\left(r_{12}\right)=\frac{1}{\pi} \sum_{n=0}^{\infty} \frac{\Gamma\left(n+\frac{1}{2}\right)^{2}}{n !^{2}}\left[A_{n}(r)-B_{n}(r)\right]
$$

where

$$
A_{n}(r)= \begin{cases}\frac{2}{5} r^{2}-2 r^{2} \ln r, & n=1 \\ \frac{(4 n+1)}{(n-1)(2 n+3)} r^{2}-\frac{1}{(n-1)} r^{2 n}, & n \neq 1,\end{cases}
$$

and

$$
B_{n}(r)= \begin{cases}\frac{1}{3} r^{2}-r^{2} \ln r, & n=0 \\ \frac{(2 n+1)(4 n+3)}{2 n(n+1)(2 n+3)} r^{2}-\frac{(2 n+1)}{2 n(n+1)} r^{2 n+2}, & n \neq 0 .\end{cases}
$$

This result is implicit in an earlier work of Bray and Humayun [21], who focused upon the singular part of this function. In the limit $r \rightarrow 0$, the singular terms in $C(r)$ arise from $A_{1}(r)$ and $B_{0}(r)$, and can be computed as

$$
C_{\text {sing }}\left(r_{12}\right)=\frac{1}{2} r^{2} \ln r
$$

which gives rise to a power-law tail in the structure factor $S(k) \simeq 4 \pi L^{2}(k L)^{-4}$, a result referred to as the "generalized Porod law', $[22,15]$.

\section{B. Case with $\beta \neq 0$}

We would like to undertake a similar asymptotic analysis in the general case with $\beta \neq 0$. As we are interested only in the limit $r \rightarrow 0$, we again discard the step function on the RHS of Eq. (9). In that case, we obtain

$$
\begin{aligned}
C\left(r_{12}\right)= & \frac{\left(1-q^{2}\right)}{\pi} \operatorname{Re} \sum_{n=0}^{\infty} \frac{(i q L)^{n}}{n !} \int_{0}^{1} d x x e^{-i q L x} \\
& \times \int_{0}^{2 \pi} d \theta(x+r \cos \theta)\left(x^{2}+r^{2}+2 x r \cos \theta\right)^{(n-1) / 2} .
\end{aligned}
$$

We will separately consider the cases with $n$ odd and $n$ even.

(a) $n$ odd. We designate $n=2 p+1$ and consider the angular integral on the RHS of Eq. (15):

$$
\begin{aligned}
\widetilde{I}_{2 p+1}(x, r)= & \int_{0}^{2 \pi} d \theta(x+r \cos \theta)\left(x^{2}+r^{2}+2 x r \cos \theta\right)^{p} \\
= & 2 x r^{2 p} \int_{0}^{\pi} d \theta\left(1+\frac{x^{2}}{r^{2}}+\frac{2 x}{r} \cos \theta\right)^{p} \\
& +2 r^{2 p+1} \int_{0}^{\pi} d \theta \cos \theta\left(1+\frac{x^{2}}{r^{2}}+\frac{2 x}{r} \cos \theta\right)^{p} \\
\equiv & 2 x r^{2 p} I_{1}+2 r^{2 p+1} I_{2} .
\end{aligned}
$$

The integrals $I_{1}$ and $I_{2}$ are obtained from Gradshteyn and Ryzhik [23], and the consolidated result is

$$
\begin{aligned}
\tilde{I}_{2 p+1}(x, r)= & 2 \pi\left[\sum_{k=0}^{p}\left(\begin{array}{l}
p \\
k
\end{array}\right)^{2} x^{2 k+1} r^{2(p-k)}+r^{2} \sum_{k=0}^{[(p-1) / 2]}\left(\begin{array}{l}
p \\
k
\end{array}\right)\right. \\
& \left.\times\left(\begin{array}{c}
p+k \\
k+1
\end{array}\right) x^{2 k+1} r^{2 k}\left(x^{2}+r^{2}\right)^{p-2 k-1}\right]
\end{aligned}
$$

where $[y]$ refers to the integer part of $y$. The corresponding contribution to $C\left(r_{12}\right)$ is

$$
\begin{aligned}
C_{1}\left(r_{12}\right)= & \frac{1-q^{2}}{\pi} \sum_{n=1,3,5, \ldots}^{\infty}(-1)^{(n-1) / 2} \frac{(q L)^{n}}{n !} \\
& \times \int_{0}^{1} d x x \sin (q L x) \widetilde{I}_{n}(x, r) .
\end{aligned}
$$

The important feature here is that the above expression for $C_{1}\left(r_{12}\right)$ contains only powers of $r^{2}$. Therefore, the overall contribution to $C\left(r_{12}\right)$ from this set of terms is analytic as $r \rightarrow 0$. In the limiting case $q=0(\beta=0)$, the above contribution is identically 0 . 
(b) $n$ even. Next, let us consider the case with $n$ even. We designate $n=2 p$, and the angular integral on the RHS of Eq. (15) is

$$
\widetilde{I}_{2 p}(x, r)=\int_{0}^{2 \pi} d \theta(x+r \cos \theta)\left(x^{2}+r^{2}+2 x r \cos \theta\right)^{(2 p-1) / 2} .
$$

We introduce $\rho_{<}=\min (x, r)$ and $\rho_{>}=\max (x, r)$ to obtain

$$
\begin{aligned}
\widetilde{I}_{2 p}(x, r)= & 2 \rho_{>}^{2 p-1} \int_{0}^{\pi} d \theta(x+r \cos \theta) \\
& \times\left(1+\frac{\rho_{<}^{2}}{\rho_{>}^{2}}+\frac{2 \rho_{<}}{\rho_{>}} \cos \theta\right)^{(2 p-1) / 2} \\
\equiv & 2 \rho_{>}^{2 p-1}\left(x I_{3}+r I_{4}\right) .
\end{aligned}
$$

The integrals $I_{3}$ and $I_{4}$ can be computed in terms of hypergeometric functions as follows [23]:

$$
I_{3}=\pi F\left(\frac{1}{2}-p, \frac{1}{2}-p ; 1 ; \frac{\rho_{<}^{2}}{\rho_{>}^{2}}\right)
$$

and

$$
\begin{aligned}
I_{4}= & \pi\left(\frac{1}{2}+p\right) \frac{\rho_{<}}{\rho_{>}} F\left(\frac{1}{2}-p, \frac{1}{2}-p ; 2 ; \frac{\rho_{<}^{2}}{\rho_{>}^{2}}\right) \\
& -\pi \frac{\rho_{<}}{\rho_{>}} F\left(\frac{1}{2}-p, \frac{1}{2}-p ; 1 ; \frac{\rho_{<}^{2}}{\rho_{>}^{2}}\right) \\
= & \pi\left(p-\frac{1}{2}\right) \frac{\rho_{<}}{\rho_{>}} F\left(\frac{3}{2}-p, \frac{1}{2}-p ; 2 ; \frac{\rho_{<}^{2}}{\rho_{>}^{2}}\right) .
\end{aligned}
$$

We have simplified Eq. (22) using the standard identity [24]

$$
\begin{gathered}
(c-a-1) F(a, b ; c ; z)+a F(a+1, b ; c ; z) \\
=(c-1) F(a, b ; c-1 ; z),
\end{gathered}
$$

with $a=1 / 2-p, b=1 / 2-p$, and $c=2$.

Combining the expressions for $I_{3}$ and $I_{4}$, we obtain

$$
\begin{aligned}
\widetilde{I}_{2 p}(x, r)= & 2 \pi \rho_{>}^{2 p-1}\left[x F\left(\frac{1}{2}-p, \frac{1}{2}-p ; 1 ; \frac{\rho_{<}^{2}}{\rho_{>}^{2}}\right)\right. \\
& \left.+r \frac{\rho_{<}}{\rho_{>}}\left(p-\frac{1}{2}\right) F\left(\frac{3}{2}-p, \frac{1}{2}-p ; 2 ; \frac{\rho_{<}^{2}}{\rho_{>}^{2}}\right)\right] .
\end{aligned}
$$

The corresponding terms in the correlation function are

$$
\begin{aligned}
C_{2}\left(r_{12}\right)= & \left(1-q^{2}\right) \sum_{n=0,2,4, \ldots}^{\infty}(-1)^{n / 2} \frac{(q L)^{n}}{n !} \int_{0}^{1} d x x \cos (q L x) \\
& \times\left[2 x \rho_{>}^{n-1} F\left(\frac{1-n}{2}, \frac{1-n}{2} ; 1 ; \frac{\rho_{<}^{2}}{\rho_{>}^{2}}\right)\right. \\
& \left.+r \rho_{>}^{n-2} \rho_{<}(n-1) F\left(\frac{3-n}{2}, \frac{1-n}{2} ; 2 ; \frac{\rho_{<}^{2}}{\rho_{>}^{2}}\right)\right] \\
\equiv & \left(1-q^{2}\right)\left(T_{1}+T_{2}\right) .
\end{aligned}
$$

The singular contributions to $C\left(r_{12}\right)$ as $r \rightarrow 0$ arise entirely from $C_{2}\left(r_{12}\right)$, as $C_{1}\left(r_{12}\right)$ is analytic in $r$. A considerable amount of algebra is involved in extracting the singular terms in $T_{1}$ and $T_{2}$. For the sake of brevity, we will sketch only the broad features of the calculation here. We have

$$
\begin{aligned}
T_{1}= & 2 \sum_{p=0}^{\infty}(-1)^{p} \frac{(q L)^{2 p}}{(2 p) ! \Gamma\left(\frac{1}{2}-p\right)^{2}} \\
& \times \sum_{m=0}^{\infty} \frac{\Gamma\left(\frac{1}{2}-p+m\right)^{2}}{m !^{2}} \int_{0}^{1} d x \cos (q L x) x^{2} \frac{\rho_{<}^{2 m}}{\rho_{>}^{2(m-p)+1}},
\end{aligned}
$$

where we have used the standard expansion for the hypergeometric function [24]. The integral on the RHS of Eq. (26) can be written as

$$
\begin{aligned}
I_{5}= & \frac{1}{r^{2(m-p)+1}} \int_{0}^{r} d x \cos (q L x) x^{2 m+2} \\
& +r^{2 m} \int_{r}^{1} d x \cos (q L x) x^{-2(m-p)+1} .
\end{aligned}
$$

The first term on the RHS of Eq. (27) is analytic as $r \rightarrow 0$. The second term contributes singular terms only if $m \geqslant p$ +1 , yielding the result

$$
I_{5}=(-1)^{m-p} \frac{(q L)^{2(m-p-1)}}{[2(m-p-1)] !} r^{2 m} \ln r+(\text { analytic terms }) .
$$

Replacing this in the expression for $T_{1}$, some algebra yields

$$
\begin{aligned}
T_{1}= & \sum_{p=0}^{\infty} \sum_{m=0}^{\infty}(-1)^{p+m+1} \frac{(q L)^{2(p+m)}}{(2 p) !(2 m) !} \\
& \times \frac{\Gamma\left(\frac{3}{2}+m\right)^{2}}{\Gamma\left(\frac{1}{2}-p\right)^{2}(m+p+1) !^{2}} r^{2(m+p+1)} \ln r \\
& + \text { (analytic terms). }
\end{aligned}
$$

A similar analysis for $T_{2}$ yields 


$$
\begin{aligned}
T_{2}= & \sum_{p=0}^{\infty} \sum_{m=0}^{\infty}(-1)^{p+m} \frac{(q L)^{2(p+m)}}{(2 p) !(2 m) !} \\
& \times \frac{\Gamma\left(\frac{1}{2}+m\right)^{2}}{\Gamma\left(\frac{1}{2}-p\right)^{2}(m+p) !^{2}} \frac{(2 m+1)}{(m+p+1)} r^{2(m+p+1)} \ln r \\
& +(\text { analytic terms }) .
\end{aligned}
$$

We can combine the singular terms from $T_{1}$ and $T_{2}$ to obtain the singular part of $C\left(r_{12}\right)$ as follows:

$$
\begin{aligned}
C_{\text {sing }}\left(r_{12}\right)= & \frac{1}{2} \sum_{p=0}^{\infty} \sum_{m=0}^{\infty}(-1)^{p+m} \frac{(q L)^{2(p+m)}}{(2 p) !(2 m) !} \\
& \times \frac{\Gamma\left(\frac{1}{2}+m\right)^{2}}{\Gamma\left(\frac{1}{2}-p\right)^{2}(m+p+1) !^{2}} \\
& \times(2 m+1)(2 p+1) r^{2(m+p+1)} \ln r .
\end{aligned}
$$

We notice that the leading-order singularity is unchanged and continues to be $C_{\text {sing }}\left(r_{12}\right) \simeq \frac{1}{2} r^{2} \ln r$, as in the case with $\beta=0$. However, there is now a sequence of subdominant singularities proportional to $(q L)^{2} r^{4} \ln r,(q L)^{4} r^{6} \ln r$, etc., and these become increasingly important as the length scale $L$ increases. These subdominant terms in $C_{\text {sing }}\left(r_{12}\right)$ are reminiscent of the leading-order singularities in models with $O(n)$ symmetry, where $n$ is even $[8,21]$. Of course, in the context of $O(n)$ models, these singularities arise only for $n$ $\leqslant d$ as there are no topological defects unless this condition is satisfied. In the present context, all these terms are already present for $d=2$. The implication for the structure-factor tail is a sequence of power-law decays with $S(k)$ $\sim(q L)^{2(m-1)} L^{d} /(k L)^{d+2 m}$, where $m=1,2$, etc. Thus, although the true asymptotic behavior in $d=2$ is still the generalized Porod tail $S(k) \sim L^{2}(k L)^{-4}$, it may be difficult to disentangle this from other power-law decays.

The results presented in this section are of relevance in determining the small-distance behavior of the correlation function, or the large-wave-vector behavior of the structure factor. This is because small length scales only probe individual defects. Nevertheless, as our forthcoming numerical results will demonstrate, the single-spiral correlation function agrees with the correlation function for multispiral morphologies (obtained numerically) over a considerable range of distances. For even larger length scales, we have to explicitly account for the modulation of the order parameter at defect-defect boundaries. We address this problem in the next section of this paper.

\section{UTILITY OF GAUSSIAN AUXILIARY FIELD ANSATZ FOR A MULTISPIRAL MORPHOLOGY}

The evolution in Fig. 1 is characterized by a morphology with multiple spirals and antispirals. Initially, spirals and an- tispirals are attracted to each other and annihilate, thereby decreasing the defect density and increasing the interdefect distance (or characteristic length scale). When the defect density is large, the spiral sizes are small and spirals are similar to vortices. Therefore, we expect an initial coarsening regime which is analogous to that for the $X Y$ model-in terms of both the domain growth law $L(t) \sim(t / \ln t)^{1 / 2}$ [25] and the morphology as characterized by the correlation function $[11,15]$. This is in accordance with our numerical simulations, as we will discuss in a future paper. Distinctive effects of spirals are seen for length scales $L>L_{c}$, where $L_{c}$ $\sim q^{-1}$ — clearly, $L_{c} \rightarrow \infty$ as $q \rightarrow 0$ (or $\beta \rightarrow 0$ ). Furthermore, there is a repulsive spiral-antispiral potential beyond a certain distance, which prevents the annealing of all defects $[4,20]$. Thus, the evolving system "freezes" (in a statistical sense) into a multispiral morphology. This should be contrasted with the case of the dynamical $X Y$ model $[\alpha=\beta$ $=0$ in Eq. (1)], where we expect the zero-temperature system to continue coarsening as $t \rightarrow \infty$.

A common theme in the characterization of dynamical evolution with a nonconserved order parameter is the introduction of a Gaussian auxiliary field $[8,10,15,26]$. Essentially, the GAF ansatz takes the form $\psi(\vec{r}, t)=F[m(\vec{r}, t)]$, where the function $F[m]$ is determined from the defect structure, and the complex field $m$ (which measures the location relative to the defect core) is assumed to obey a Gaussian distribution. The zero crossings of the field $m$ correspond to the location of defect cores. The GAF ansatz enables a straightforward computation of the correlation function for the field $\psi(\vec{r}, t)$. However, the analytical justification for the GAF ansatz is meager and its primary virtue appears to be that it works rather well in some situations [8].

Let us examine the utility of the GAF ansatz in the present context. The appropriate form of the ansatz for the CGL equation in the regime where the spiral structures are well developed is (using Hagan's solution for the spiral defect)

$$
\psi(\vec{r}, t) \simeq \frac{\sqrt{1-q^{2}} m(\vec{r}, t)}{\sqrt{1-q^{2}+|m(\vec{r}, t)|^{2}}} \exp \{-i[\omega t+q|m(\vec{r}, t)|]\},
$$

where $\omega=\beta\left(1-q^{2}\right)$; and we take $|\psi| \simeq|m|$ near the defect core $(|m| \rightarrow 0)$, in accordance with Hagan's solution. The field $m\left(=m_{1}+i m_{2}\right)$ is assumed to obey a Gaussian distribution with

$$
P\left(m_{i}\right)=\frac{1}{\sqrt{2 \pi \sigma^{2}}} \exp \left(-\frac{m_{i}^{2}}{2 \sigma^{2}}\right), \quad i=1,2,
$$

where $\sigma^{2}=\left\langle m_{i}(\vec{r}, t)^{2}\right\rangle$; and the fields $m_{1}(\vec{r}, t)$ and $m_{2}(\vec{r}, t)$ are taken to be statistically independent of each other.

Our numerical results show that the GAF ansatz in Eq. (32) is reasonable in the vicinity of defects. However, it is inappropriate for defect-defect boundaries, where the orderparameter amplitude $|\psi|$ is often larger than $\sqrt{1-q^{2}}$. This is demonstrated in Fig. 4, which replots Fig. 1 with defect lo- 

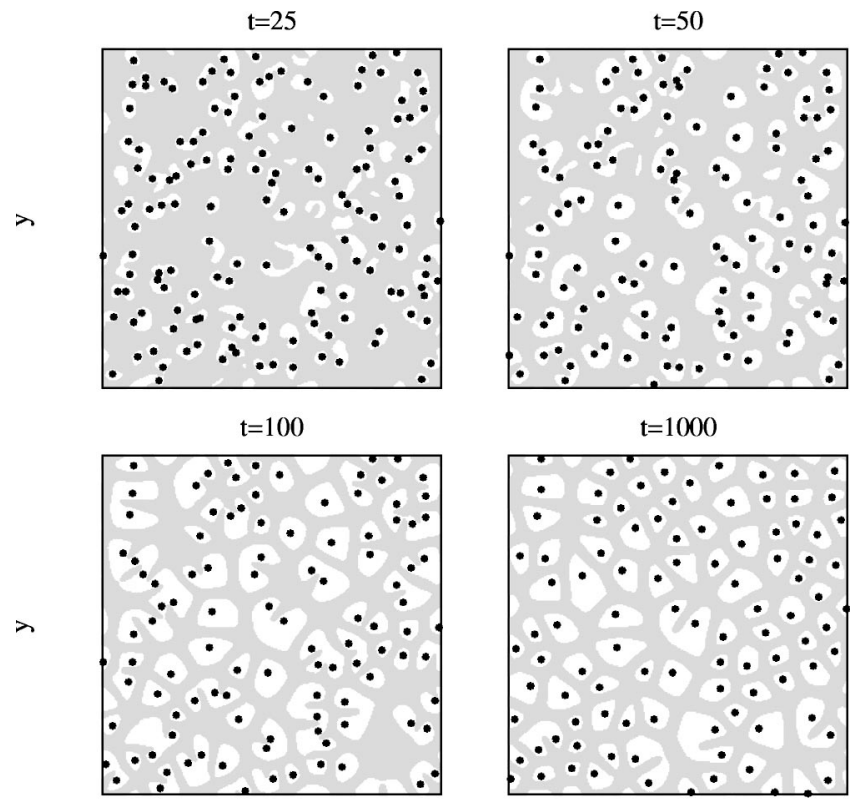

$\mathrm{x}$

$$
\begin{aligned}
P^{\prime}\left(m_{1}^{\prime}\right)= & \frac{1}{2 \pi \sigma^{2}} \int_{0}^{\infty} d|m||m| \exp \left(-\frac{|m|^{2}}{2 \sigma^{2}}\right) \\
& \times \int_{0}^{2 \pi} d \theta \delta\left(m_{1}^{\prime}-|m| \cos (\theta-\phi)\right) .
\end{aligned}
$$

Because of the periodicity of the function $\cos (\theta-\phi)$, the phase factor $\phi$ is inconsequential and

$$
P^{\prime}\left(m_{1}^{\prime}\right)=\frac{1}{\sqrt{2 \pi \sigma^{2}}} \exp \left(-\frac{m_{1}^{\prime 2}}{2 \sigma^{2}}\right),
$$

and a similar distribution also applies for the variable $m_{2}^{\prime}$.

Thus, we have the appropriate GAF ansatz (dropping primes) as follows:

$$
\psi(\vec{r}, t)=\frac{\sqrt{1-q^{2}} m(\vec{r}, t)}{\sqrt{1-q^{2}+|m(\vec{r}, t)|^{2}}},
$$

where the variables $m_{1}(\vec{r}, t)$ and $m_{2}(\vec{r}, t)\left[m(\vec{r}, t)=m_{1}(\vec{r}, t)\right.$ $\left.+i m_{2}(\vec{r}, t)\right]$ are Gaussian and independent of each other. The inverse relation between the variables $\psi$ and $m$ is

$$
m(\vec{r}, t)=\frac{\sqrt{1-q^{2}} \psi(\vec{r}, t)}{\sqrt{1-q^{2}-|\psi(\vec{r}, t)|^{2}}} .
$$

cations marked by asterisks and regions where $|\psi|$ $>\sqrt{1-q^{2}}$ marked in gray. As discussed before, for early times (e.g., $t=25$ ), the system evolution is governed by the interaction of vortices. Thus, the appropriate GAF ansatz should have $\max (|\psi|)=1$, as in the case of the $X Y$ model. For late times (e.g., $t=1000$ ), the system has well-developed spirals. Nevertheless, the GAF ansatz for the order-parameter field is obviously inappropriate for large regions of space at these parameter values. For other values of $\beta$, the same general arguments apply although there are changes in the crossover time to spiral-mediated growth, and the fraction of spatial region where the GAF ansatz is unreasonable.

Let us examine the validity of the GAF ansatz in regions where $|\psi|<\sqrt{1-q^{2}}$. We can simplify the ansatz in Eq. (32) by defining the variable $m^{\prime}=m e^{-i \phi}$, where $\phi=\omega t+q|m|$. Then we have the corresponding probability distribution for (say) $m_{1}^{\prime}$ as

$$
\begin{aligned}
P^{\prime}\left(m_{1}^{\prime}\right)= & \int_{-\infty}^{\infty} d m_{1} \int_{-\infty}^{\infty} d m_{2} \delta\left(m_{1}^{\prime}-m_{1} \cos \phi\right. \\
& \left.-m_{2} \sin \phi\right) P\left(m_{1}\right) P\left(m_{2}\right) \\
= & \frac{1}{2 \pi \sigma^{2}} \int_{-\infty}^{\infty} d m_{1} \int_{-\infty}^{\infty} d m_{2} \delta\left(m_{1}^{\prime}-m_{1} \cos \phi\right. \\
& \left.-m_{2} \sin \phi\right) \exp \left(-\frac{m_{1}^{2}+m_{2}^{2}}{2 \sigma^{2}}\right)
\end{aligned}
$$

As usual, we transform $\left(m_{1}, m_{2}\right) \rightarrow(|m|, \theta)$ to obtain

We want to examine the validity of the GAF ansatz numerically $[27,28]$ in the context of the evolution depicted in Fig. 1 (or Fig. 4). The appropriate parameter values are $\beta$ $=1$ and $q \simeq 0.306$ [18] (see Fig. 2). In Fig. 5, we plot the single-variable distribution for the field $m_{1}(\vec{r}, t)$, obtained directly from our simulation of the CGL equation using Eq. (38) in regions where $|\psi|<\sqrt{1-q^{2}}$. The data in Fig. 5 are obtained as an average over five independent runs for $N^{2}$ lattices, with $N=512$. (Details of our simulation will be provided in a subsequent paper II.) Figure 5(a) is a plot of $P\left(m_{1}\right)$ vs $m_{1}$ from four different times-corresponding to the evolution pictures shown in Fig. 1. In Fig. 5(b), we have scaled variables and superposed the data for $P\left(m_{1}\right) \sigma$ vs $m_{1} / \sigma$, where $\sigma$ is obtained from the best fit of the numerical data to the functional form in Eq. (36). The data collapse onto a single master curve, which is reasonably approximated by the Gaussian form $P(x)=(1 / \sqrt{2 \pi}) e^{-x^{2} / 2}$, denoted as a solid line in Fig. 5(b).

Figure 5 was obtained by focusing only on regions where $|\psi|<\sqrt{1-q^{2}}$, which is essentially equivalent to considering disjoint spirals, for which the correlation function has already been obtained in Sec. II. We have examined various ad hoc methods of improving the GAF ansatz in Eq. (37). For example, one could set the saturation amplitude of the order parameter to its maximum value $\left(|\psi|_{\text {sat }} \simeq 1\right.$ for Fig. 1), rather than $|\psi|_{\text {sat }}=\sqrt{1-q^{2}}$. Figure 6 plots the resultant probability distributions $P\left(m_{1}\right)$ vs $m_{1}$ with $|\psi|_{\text {sat }}=1$. For early times $(t=25)$, the distribution has a Gaussian form, as expected from our analogy with the $X Y$ model. However, with the 

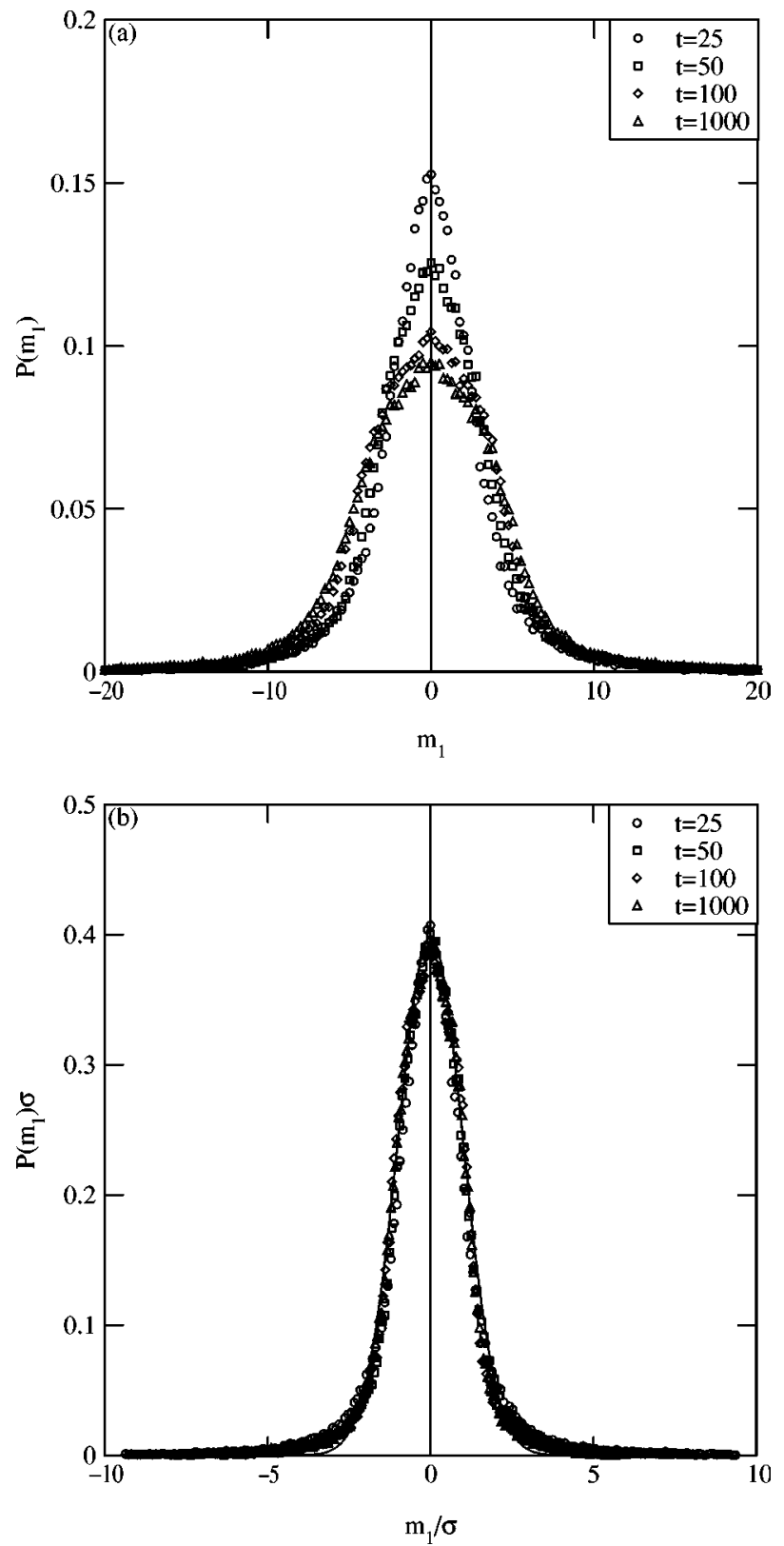

FIG. 5. (a) Plot of data for $P\left[m_{1}(\vec{r}, t)\right]$ vs $m_{1}(\vec{r}, t)$ from four different times $t=25,50,100,1000$-denoted by the symbols shown. The parameter values are identical to those in Fig. 1. We use Eq. (38) to obtain data for $m_{1}(\vec{r}, t)$ directly from the order-parameter field in our numerical solution of the CGL equation-considering only regions where $|\psi|<\sqrt{1-q^{2}}$. The data were obtained as an average over five independent runs for $N^{2}$ lattices $(N=512)$. (b) Scaled plot of data from (a). We superpose data for $P\left[m_{1}(\vec{r}, t)\right] \sigma(t)$ vs $m_{1}(\vec{r}, t) / \sigma(t)$, where $\sigma(t)$ is obtained from the best fit of the numerical data to a Gaussian distribution. The solid line refers to the Gaussian function $P(x)=(1 / \sqrt{2 \pi}) e^{-x^{2} / 2}$.

emergence of well-formed spirals, the distribution develops a double peak and is clearly non-Gaussian.

We have also studied some other possible ways of rectifying the GAF ansatz. We find that these ad hoc approaches invariably result in non-Gaussian distributions for the auxiliary field. Perhaps a more honest approach should be based

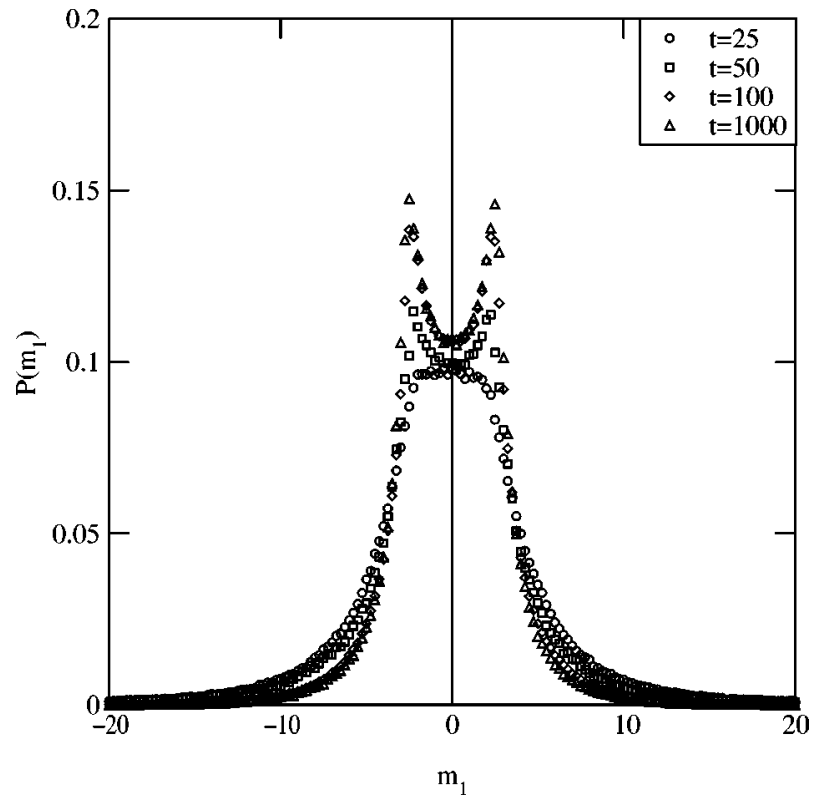

FIG. 6. Plot of data for $P\left[m_{1}(\vec{r}, t)\right]$ vs $m_{1}(\vec{r}, t)$ from times $t$ $=25,50,100,1000$ - denoted by the symbols shown. The parameter values and statistical details are identical to those for Fig. 5(a). Data for $m_{1}(\vec{r}, t)$ are obtained directly from the numerical data for $\psi(\vec{r}, t)$, using Eq. (37) with $|\psi|_{\text {sat }}=\sqrt{1-q^{2}}$ replaced by $|\psi|_{\text {sat }}=1$.

on the order-parameter field for spiral-spiral pairs [20] as a function of two independent auxiliary fields-referring to distances from the centers of the two spirals. We are presently studying the utility of such an approach for characterizing multispiral morphology.

\section{SUMMARY AND DISCUSSION}

Let us conclude this paper with a brief summary and discussion of our results. We have undertaken a detailed analytical and numerical investigation of nonequilibrium dynamics in a special case of the complex Ginzburg-Landau equation. Our results are described in a two-stage exposition. This paper constitutes the first stage of this exposition, and describes analytical results for the time-dependent correlation function. Our analytical arguments rely on the significance of spiral-defect structures in determining the morphology and evolution of the CGL equation from a random initial condition.

In this paper, we describe results for the exact correlation function $C\left(r_{12}\right)$ of a single spiral defect of size $L$, and undertake its asymptotic analysis in the limit $r_{12} / L \rightarrow 0$ but $r_{12} / \xi \gg 1$, where $\xi$ is the size of the defect core. We find that there is a sequence of singularities in this limit, which are reminiscent of singularities for defects with $O(n)$ symmetry, where $n$ is even. However, the dominant singularity as $r_{12} / L \rightarrow 0$ corresponds to the case of vortex defects, as expected. The implications for the large-wave-vector tail of the structure factor are also discussed.

We also investigate the validity of the Gaussian auxiliary field ansatz in the context of multispiral morphologies. For early times ( $L<L_{c} \sim q^{-1}$ ), domain growth in the CGL equa- 
tion is analogous to that for the $X Y$ model, whose domain growth law and correlation function are well understood [8]. For later times, we find that the simple GAF ansatz is not reasonable, as it is unable to account for order-parameter modulations in the defect-defect boundaries. We have attempted ad-hoc improvements of the GAF ansatz but these invariably result in non-Gaussian distributions for the corresponding auxiliary field. We are presently investigating the possibility of formulating a generalized GAF ansatz in terms of the order-parameter field for a spiral-spiral pair.

More generally, the utility of the GAF ansatz arises from the summation over phases from many defects, which results in a near-Gaussian distribution for the auxiliary field. However, in the present context, the shocks between spirals effectively isolate one spiral region from the influence of other regions. As a matter of fact, the waves from other spirals decay exponentially through the shock and the phase of a point is always dominated by the nearest spiral. Therefore, we expect that the correlation function will be dominated by the single-spiral result-in accordance with our numerical results.
In a future paper we will present detailed numerical results for phase-ordering dynamics in the CGL equation. In particular, we will focus upon the crossover from vortexmediated dynamics (at early times) to spiral-mediated dynamics (at late times). Furthermore, we will compare our numerical results for the correlation function of the orderparameter field with the analytic form for a single-spiral defect presented in this paper.

Before we conclude this paper, it is worth stressing that the results presented are easily adaptable to the general case of the CGL equation with $\alpha, \beta \neq 0$. Again, the evolving morphology in a large region of parameter space is characterized by the presence and annihilation of spirals and antispirals [6]. The results of the present paper apply directly in that case also, with minor modifications in the functional form of the spiral solution in Sec. II.

\section{ACKNOWLEDGMENTS}

S.P. is grateful to A. J. Bray and H. Chate for useful discussions. S.K.D. is grateful to the University Grants Commission, India, for financial support.
[1] For example, see S. C. Müller, T. Plesser, and B. Hess, Physica D 24, 71 (1987); G. S. Skinner and H. L. Swinney, ibid. 48, 1 (1991).

[2] R. W. Walden, P. Kolodner, A. Passner, and C. M. Surko, Phys. Rev. Lett. 55, 496 (1985); E. Moses and V. Steinberg, Phys. Rev. A 34, 693 (1986).

[3] F. T. Arecchi, G. Giacomelli, P. L. Ramazza, and S. Residori, Phys. Rev. Lett. 65, 2531 (1990); 67, 3749 (1991).

[4] M. C. Cross and P. C. Hohenberg, Rev. Mod. Phys. 65, 851 (1993).

[5] For example, see Y. Kuramoto, Chemical Oscillations, Waves and Turbulence (Springer-Verlag, Berlin, 1984); W. van Saarloos, in Spatiotemporal Patterns in Nonequilibrium Systems, edited by P. E. Cladis and P. Palffy-Muhoray (AddisonWesley, Reading, MA, 1994).

[6] For example, see H. Chate, Nonlinearity 7, 185 (1994) for the $d=1$ CGL equation; H. Chate and P. Manneville, Physica A 224, 348 (1996) for the $d=2$ CGL equation.

[7] K. Binder, in Materials Science and Technology, Vol. 5: Phase Transformations of Materials, edited by R. W. Cahn, P. Haasen, and E. J. Kramer (VCH, Weinheim, 1991), p. 405.

[8] A. J. Bray, Adv. Phys. 43, 357 (1994).

[9] I. M. Lifshitz, Sov. Phys. JETP 15, 939 (1962); S. M. Allen and J. W. Cahn, Acta Metall. 27, 1085 (1979).

[10] T. Ohta, D. Jasnow, and K. Kawasaki, Phys. Rev. Lett. 49, 1223 (1982).

[11] S. Puri, Phys. Lett. A 164, 211 (1992).

[12] M. Suzuki, Prog. Theor. Phys. 56, 477 (1976).

[13] K. Kawasaki, M. C. Yalabik, and J. D. Gunton, Phys. Rev. A 17, 455 (1978).
[14] S. Puri and C. Roland, Phys. Lett. A 151, 500 (1990).

[15] A. J. Bray and S. Puri, Phys. Rev. Lett. 67, 2670 (1991).

[16] H. Toyoki, Phys. Rev. B 45, 1965 (1992).

[17] A. J. Bray and A. D. Rutenberg, Phys. Rev. E 49, R27 (1994).

[18] P. S. Hagan, SIAM (Soc. Ind. Appl. Math.) J. Appl. Math. 42, 762 (1982).

[19] I. S. Aranson, L. B. Aranson, L. Kramer, and A. Weber, Phys. Rev. A 46, R2992 (1992); A. Weber, L. Kramer, I. S. Aranson, and L. B. Aranson, Physica D 61, 279 (1992).

[20] I. S. Aranson, L. Kramer, and A. Weber, Phys. Rev. E 47, 3231 (1993).

[21] A. J. Bray and K. Humayun, Phys. Rev. E 47, R9 (1993).

[22] G. Porod, in Small-Angle X-Ray Scattering, edited by O. Glatter and O. Kratky (Academic Press, New York, 1982); Y. Oono and S. Puri, Mod. Phys. Lett. B 2, 861 (1988).

[23] I. S. Gradshteyn and I. M. Ryzhik, in Table of Integrals, Series, and Products, edited by A. Jeffrey (Academic Press, London, 1994).

[24] Handbook of Mathematical Functions, edited by M. Abramowitz and I. Stegun (Dover, New York, 1968).

[25] A. N. Pargellis, P. Finn, J. W. Goodby, P. Panizza, B. Yurke, and P. E. Cladis, Phys. Rev. A 46, 7765 (1992); B. Yurke, A. N. Pargellis, T. Kovacs, and D. A. Huse, Phys. Rev. E 47, 1525 (1993).

[26] G. F. Mazenko, Phys. Rev. Lett. 63, 1605 (1989); Phys. Rev. B 42, 4487 (1990); 43, 5747 (1991).

[27] C. Yeung, Y. Oono, and A. Shinozaki, Phys. Rev. E 49, 2693 (1994).

[28] J. Das and M. Rao, Phys. Rev. E 57, 5069 (1998). 